\title{
UNCERTAINTY PRINCIPLES CONNECTED WITH THE MÖBIUS INVERSION FORMULA
}

\author{
PAUL POLLACK ${ }^{\otimes}$ and CARLO SANNA
}

(Received 29 October 2012; accepted 3 November 2012; first published online 25 January 2013)

\begin{abstract}
Two arithmetic functions $f$ and $g$ form a Möbius pair if $f(n)=\sum_{d \mid n} g(d)$ for all natural numbers $n$. In that case, $g$ can be expressed in terms of $f$ by the familiar Möbius inversion formula of elementary number theory. In a previous paper, the first-named author showed that if the members $f$ and $g$ of a Möbius pair are both finitely supported, then both functions vanish identically. Here we prove two significantly stronger versions of this uncertainty principle. A corollary is that in a nonzero Möbius pair, one cannot have both $\sum_{f(n) \neq 0} 1 / n<\infty$ and $\sum_{g(n) \neq 0} 1 / n<\infty$.
\end{abstract}

2010 Mathematics subject classification: primary 11A25; secondary 11N37.

Keywords and phrases: Möbius inversion, Möbius transform, uncertainty principle, sets of multiples.

\section{Introduction}

Let $f$ be an arithmetic function, that is, a function from the set of natural numbers (positive integers) to the complex numbers. The Dirichlet transform $\hat{f}$ and the Möbius transform $\check{f}$ of $f$ are defined by the equations

$$
\hat{f}(n):=\sum_{d \mid n} f(d) \quad \text { and } \quad \check{f}(n):=\sum_{d \mid n} \mu\left(\frac{n}{d}\right) f(d) .
$$

In a first course in number theory, one learns (Möbius inversion) that the Dirichlet and Möbius transforms are inverses of each other, that is,

$$
f=\check{\hat{f}}=\hat{\tilde{f}}
$$

for every $f$. In a short note [Pol11], the first author gave a simple proof of the following uncertainty principle for the Möbius transform: if $f$ is an arithmetic function, not identically zero, then the support of $f$ and the support of $\breve{f}$ cannot both be finite. (Here the support of an arithmetic function $h$ refers to the set $\{n: h(n) \neq 0\}$. We denote this by $\operatorname{supp}(h))$ In this note, we present two substantial quantitative strengthenings of this result.

(c) 2013 Australian Mathematical Publishing Association Inc. 0004-9727/2013 \$16.00 
Call a pair of functions $(f, g)$ a Möbius pair if $f=\hat{g}$ (equivalently, if $g=\check{f}$ ). For the sake of typography, we state our results in terms of $f$ and $g$ rather than $f$ and $\check{f}$.

Theorem 1.1. Suppose that $(f, g)$ is a nonzero Möbius pair. If

$$
\sum_{n \in \operatorname{supp}(g)} \frac{1}{n}<\infty,
$$

then $\operatorname{supp}(f)$ possesses a positive asymptotic density. The same result holds with the roles of $f$ and $g$ reversed.

Our second result is similar, but now weighted by the absolute values of $f$ and $g$. Recall that the mean value of an arithmetic function $h$ is the limit, as $x \rightarrow \infty$, of the finite averages $(1 / x) \sum_{n \leq x} h(n)$.

Theorem 1.2. Suppose that $(f, g)$ is a nonzero Möbius pair. If

$$
\sum_{n=1}^{\infty} \frac{|g(n)|}{n}<\infty
$$

then $|f|$ possesses a nonzero mean value. The same result holds with the roles of $f$ and $g$ reversed.

REMARK 1.3. Our Theorem 1.2 may be compared with a classical theorem of Wintner (see [Win43, p. 20]), according to which $\sum_{n=1}^{\infty}|g(n)| / n<\infty$ implies that $f$ has the (possibly vanishing) mean value $\sum_{n=1}^{\infty} g(n) / n$.

For the rest of this paper, call a set $\mathscr{A}$ of natural numbers thin if $\sum_{a \in \mathscr{A}} 1 / a<\infty$. One can conclude from Theorem 1.1 and partial summation (see the proof of Lemma 2.4 below) that in a nonzero Möbius pair $(f, g), \operatorname{supp}(f)$ and $\operatorname{supp}(g)$ cannot both be thin. One might wonder why we bother with thin sets instead of dealing directly with natural density. The answer is given in our final theorem, which shows that the asymptotic densities of $\operatorname{supp}(f)$ and $\operatorname{supp}(g)$ can be prescribed arbitrarily. Our notation for the density of a set $\mathscr{A} \subset \mathbf{N}$ is $\mathbf{d}(\mathscr{A})$.

Theorem 1.4. For any $\alpha, \beta \in[0,1]$, one can find a nonzero Möbius pair $(f, g)$ for which $\mathbf{d}(\operatorname{supp}(f))=\alpha$ and $\mathbf{d}(\operatorname{supp}(g))=\beta$. Moreover, $f$ and $g$ can be chosen as multiplicative functions.

Notation and conventions. Throughout, the letter $p$ is reserved for a prime variable. We continue to use $\mathbf{d}(\mathscr{A})$ for the natural density of $\mathscr{A}$, defined as the limit, as $x \rightarrow \infty$, of $(1 / x) \#\{n \in \mathscr{A}: n \leq x\}$. The lower density $\underline{\mathbf{d}}(\mathscr{A})$ and upper density $\overline{\mathbf{d}}(\mathscr{A})$ are defined analogously, with lim inf and lim sup replacing lim. We use $O$ and $o$-notation with its standard meaning. We write $p \| n$ when $p \mid n$ and $p^{2} \nmid n$. Whenever we refer to arithmetic functions $f$ and $g$, it is to be assumed that $(f, g)$ is a Möbius pair. 


\section{Preliminaries}

In this section, we collect a number of lemmas needed in later arguments. The first is a well-known sufficient condition for asymptotic density to be countably additive.

LemMa 2.1. Let $\mathscr{A}_{1}, \mathscr{A}_{2}, \mathscr{A}_{3}, \ldots$ be a sequence of disjoint sets of natural numbers, each of which possesses an asymptotic density. Suppose that $\overline{\mathbf{d}}\left(\cup_{i>k}^{\infty} \mathscr{A}_{i}\right) \rightarrow 0$ as $k \rightarrow \infty$. Then the union of the $\mathscr{A}_{i}$ has a natural density, and in fact

$$
\mathbf{d}\left(\bigcup_{i=1}^{\infty} \mathscr{A}_{i}\right)=\sum_{i=1}^{\infty} \mathbf{d}\left(\mathscr{A}_{i}\right) \text {. }
$$

Proof. The proof is easy and so we include it here. Writing $\bigcup_{i=1}^{\infty} \mathscr{A}_{i}=\left(\bigcup_{i=1}^{k} \mathscr{A}_{i}\right) \cup$ $\left(\bigcup_{i>k} \mathscr{A}_{i}\right)$, we find that for each $k$,

$$
\sum_{i=1}^{k} \mathbf{d}\left(\mathscr{A}_{i}\right) \leq \underline{\mathbf{d}}(\mathscr{A}) \leq \overline{\mathbf{d}}(\mathscr{A}) \leq\left(\sum_{i=1}^{k} \mathbf{d}\left(\mathscr{A}_{i}\right)\right)+\overline{\mathbf{d}}\left(\bigcup_{i>k} \mathscr{A}_{i}\right) .
$$

Now, letting $k \rightarrow \infty$ gives the lemma.

If $\mathscr{A}$ is any set of natural numbers, we define its set of multiples $\mathscr{M}(\mathscr{A})$ as the collection of positive integers possessing at least one divisor from $\mathscr{A}$. In other words, $\mathscr{M}(\mathscr{A}):=\{a n: a \in \mathscr{A}, n \in \mathbf{N}\}$. The next lemma collects two basic results on natural densities of sets of multiples.

Lemma 2.2. If $\mathscr{A}$ is thin, then $\mathscr{M}(\mathscr{A})$ possesses an asymptotic density. Moreover, if $1 \notin \mathscr{A}$, then $\mathbf{d}(\mathscr{M}(\mathscr{A}))<1$.

Proof. (Sketch) The existence of $\mathbf{d}(\mathscr{M}(\mathscr{A}))$ for thin sets $\mathscr{A}$ is due to Erdôs [Erd34]. It follows from an inequality of Heilbronn [Hei37] and Rohrbach [Roh37] that, whenever $\mathbf{d}(\mathscr{M}(\mathscr{A}))$ exists,

$$
\mathbf{d}(\mathscr{M}(\mathscr{A})) \leq 1-\prod_{a \in \mathscr{A}}\left(1-\frac{1}{a}\right)
$$

the second assertion of the lemma is now immediate. For more context for these results, one can consult [Ha196, Ch. 0].

According to the first half of Lemma 2.2, the set of $n$ possessing at least one divisor from a thin set $\mathscr{A}$ has an asymptotic density. The next lemma allows us to draw a similar conclusion when we prescribe exactly which members $d$ of $\mathscr{A}$ are divisors of $n$. For technical reasons which will emerge later, we formulate this result so as to allow us to also prescribe which of the quotients $n / d$ are squarefree.

Lemma 2.3. Let $\mathscr{A}$ be a thin set of positive integers. If $\mathscr{T} \subset \mathscr{S} \subset \mathscr{A}$, where $\mathscr{S}$ is finite, then the set of positive integers $n$ for which both:

(i) $\mathscr{S}=\{d \in \mathscr{A}: d \mid n\}$; and

(ii) $\mathscr{T}=\{d \in \mathscr{S}: d \mid n, \mu(n / d) \neq 0\}$,

has an asymptotic density. 
Proof. For each positive integer $n$ satisfying condition (i) above, define $\chi(n)$ by the equation

$$
\chi(n):=\prod_{d \in \mathscr{T}}|\mu(n / d)| \prod_{c \in \mathscr{S} \backslash \mathscr{T}}\left(1-\left|\mu\left(\frac{n}{c}\right)\right|\right) ;
$$

for values of $n$ not satisfying condition (i), put $\chi(n)=0$. Then $\chi$ is the indicator function of those $n$ satisfying both (i) and (ii). Moreover, when $n$ satisfies (i), expanding the final product in (2.1) reveals that

$$
\chi(n)=\sum_{\mathscr{T} \subset \mathscr{U} \subset \mathscr{S}}(-1)^{|\mathscr{U}|-|\mathscr{T}|} \prod_{e \in \mathscr{\mathscr { U }}}\left|\mu\left(\frac{n}{e}\right)\right| .
$$

So, using ' to denote a sum restricted to integers $n$ satisfying (i), we find that the count of $n \leq x$ satisfying both (i) and (ii) is given by

$$
\begin{aligned}
\sum_{n \leq x} \chi(n) & =\sum_{\mathscr{T} \subset \mathscr{U} \subset \mathscr{S}}(-1)^{|\mathscr{U}|-|\mathscr{T}|} \sum_{n \leq x}^{\prime} \prod_{e \in \mathscr{U}}\left|\mu\left(\frac{n}{e}\right)\right|, \\
& =\sum_{\mathscr{T} \subset \mathscr{U} \subset \mathscr{S}}(-1)^{|\mathscr{U}|-|\mathscr{T}|} \#\{n \leq x: n \text { satisfies (i), } n / e \text { is squarefree for all } e \in \mathscr{U}\} .
\end{aligned}
$$

Dividing by $x$ and letting $x \rightarrow \infty$, it suffices to prove that for each set $\mathscr{U}$ with $\mathscr{T} \subset \mathscr{U} \subset \mathscr{S}$, the set

$$
\mathscr{V}:=\{n \in \mathbb{N}: n \text { satisfies (i), } n / e \text { is squarefree for all } e \in \mathscr{U}\}
$$

possesses an asymptotic density.

We prove this last claim by showing that belonging to $\mathscr{V}$ amounts to not lying in the set of multiples of an appropriately constructed thin set. First, notice that in order for $n$ to satisfy (i), it is necessary that $L \mid n$, where $L:=\operatorname{lcm}\{d \in \mathscr{S}\}$. So, each of our candidate values of $n$ can be written in the form $n=L q$. For condition (i) to hold, one also needs that if $a \in \mathscr{A} \backslash \mathscr{S}$, then $a \nmid L q$; in other words, $a / \operatorname{gcd}(a, L) \nmid q$. The second condition in the definition (2.2) of $\mathscr{V}$ requires that for each $e \in \mathscr{U}$, the number $L q / e$ is not divisible by any of $2^{2}, 3^{2}, 4^{2}, \ldots$ Equivalently, $q$ cannot be divisible by any of the numbers $h^{2} / \operatorname{gcd}\left(h^{2}, L / e\right)$ with $h \geq 2$. So, for $n=L q$ to belong to the set $\mathscr{V}$, it is necessary and sufficient that $q$ does not belong to the set of multiples of

$$
\left\{\frac{a}{\operatorname{gcd}(a, L)}: a \in \mathscr{A} \backslash \mathscr{S}\right\} \cup\left(\bigcup_{e \in \mathscr{U}}\left\{\frac{h^{2}}{\operatorname{gcd}\left(h^{2}, L / e\right)}: h \geq 2\right\}\right) .
$$

Call this set $\mathscr{K}$. Then $\mathscr{K}$ is a finite union of thin sets and so is thin. (We use here that $\mathscr{A}$ is thin, by hypothesis, and that the sum of the reciprocals of the squares converges.) Hence, $\mathscr{M}(\mathscr{K})$ has a natural density, and

$$
\mathbf{d}(\mathscr{V})=\frac{\mathbf{d}(\mathbf{N} \backslash \mathscr{M}(\mathscr{K}))}{L}=\frac{1-\mathbf{d}(\mathscr{M}(\mathscr{K}))}{L} .
$$

This completes the proof. 
We also need a simple mean-value theorem of Kronecker [Kro87].

LEMMA 2.4. Let $h(n)$ be an arithmetic function. If $\sum_{n=1}^{\infty} h(n) / n$ converges, then $h$ has mean value zero.

Proof. Let $H(x):=\sum_{n \leq x} h(n)$, and let $G(x):=\sum_{n \leq x} h(n) / n$. By hypothesis, there is a constant $\rho$ so that $G(x)=\rho+o(1)$, as $x \rightarrow \infty$. Then, as $x \rightarrow \infty$,

$$
\begin{aligned}
H(x) & =x \cdot G(x)-\int_{1}^{x} G(t) d t \\
& =(\rho x+o(x))-\int_{1}^{x}(\rho+o(1)) d t=o(x) .
\end{aligned}
$$

Hence, $h$ has mean value zero.

Our final lemma, needed in the proof of Theorem 1.2, is a generalisation of the well-known result that the squarefree numbers have asymptotic density $6 / \pi^{2}$. The proof consists of easy sieving; compare with [Lan53, pp. 633-635].

LeMma 2.5. For each natural number $P$, the set of squarefree integers relatively prime to $P$ has asymptotic density

$$
\frac{6}{\pi^{2}} \prod_{p \mid P}\left(1+\frac{1}{p}\right)^{-1} .
$$

\section{Proof of Theorem 1.1}

We split the proof into two parts.

Proof that IF $g$ HAS THIN SUPPoRT, THEN $\operatorname{supp}(f)$ HAS POSITIVE DENSITY. We begin by showing that the support of $f$ has some asymptotic density. We defer showing that this density is positive to the very end of the argument.

Call two elements of $\operatorname{supp}(f)$ equivalent if they share the same set of divisors from $\operatorname{supp}(g)$. Enumerate the equivalence classes as $\mathscr{A}_{1}, \mathscr{A}_{2}, \mathscr{A}_{3}, \ldots$ Then each $\mathscr{A}_{i}$ is the set of natural numbers possessing a prescribed set $\mathscr{S}_{i}$ of divisors from $\operatorname{supp}(g)$. Each $\mathscr{S}_{i}$ is thin (as a subset of the thin set $\operatorname{supp}(g)$ ), and so Lemma 2.3 shows that each of the sets $\mathscr{A}_{i}$ possesses a natural density. (Note that the full strength of Lemma 2.3 is not required, since we are only prescribing $\mathscr{S}$, not $\mathscr{T}$.) Now,

$$
\operatorname{supp}(f)=\bigcup_{i} \mathscr{A}_{i},
$$

and the right-hand union is disjoint. If there are only finitely many classes $\mathscr{A}_{i}$, then the existence of $\mathbf{d}(\operatorname{supp}(f))$ follows immediately from finite additivity. So, suppose that there are infinitely many $\mathscr{A}_{i}$. Since only finitely many natural numbers lie below any given bound, it is clear that with

$$
m_{k}:=\min _{i>k}\left(\max _{d \in \mathscr{S}_{i}} d\right)
$$

we have $m_{k} \rightarrow \infty$ as $k \rightarrow \infty$. 
Now, if $n \in \bigcup_{i>k} \mathscr{A}_{i}$, then $\{d \mid n: d \in \operatorname{supp}(g)\}=\mathscr{S}_{i}$ for some $i>k$. Thus, $n$ has a divisor $d \in \operatorname{supp}(g)$ with $d \geq m_{k}$, and so

$$
\overline{\mathbf{d}}\left(\bigcup_{i>k} \mathscr{A}_{i}\right) \leq \sum_{\substack{d \geq m_{k} \\ d \in \operatorname{supp}(g)}} \frac{1}{d} .
$$

As $k \rightarrow \infty$, the right-hand side of this inequality tends to zero, because of the thinness of $\operatorname{supp}(g)$. So, Lemma 2.1 shows that $\mathbf{d}(\operatorname{supp}(f))=\sum_{i} \mathbf{d}\left(\mathscr{A}_{i}\right)$. This completes the proof that $\operatorname{supp}(f)$ has an asymptotic density.

We now show that $\mathbf{d}(\operatorname{supp}(f))>0$. Let $d$ be the smallest member of $\operatorname{supp}(g)$. We claim that a positive proportion of numbers $n$ have $d$ as their only divisor from $\operatorname{supp}(g)$, so that $f(n)=g(d) \neq 0$. To prove the claim, note that if $n=d m$ has a divisor from $\operatorname{supp}(g)$ other than $d$, then $m$ is a multiple of an element from the set $\{e / \operatorname{gcd}(e, d): e \neq d, e \in \operatorname{supp}(g)\}$. This set is thin $($ since $\operatorname{supp}(g)$ is) and does not contain 1 (by the minimality of $d$ ). From Lemma 2.2, we see that the corresponding values of $m$ comprise a set of density less than 1. Consequently, a positive proportion of multiples $n$ of $d$ have $d$ as their only divisor from $\operatorname{supp}(g)$, as was to be shown.

Proof that if $f$ Has thin SUPPort, Then $\operatorname{supp}(g)$ Has Positive DENSITy. This is similar to the first half. By Möbius inversion, $g(n)=\sum_{d \mid n} f(d) \mu(n / d)$. Motivated by this, we call two elements $n_{1}$ and $n_{2}$ of $\operatorname{supp}(g)$ equivalent if they share the same set of divisors $d$ from $\operatorname{supp}(f)$ and $n_{1} / d$ and $n_{2} / d$ are squarefree for the same subset of these $d$. The existence of the density of $\operatorname{supp}(g)$ then follows from an argument entirely analogous to that seen above (but now using the full strength of Lemma 2.3).

Let $d$ be the smallest element of $\operatorname{supp}(f)$. To show that $\mathbf{d}(\operatorname{supp}(g))>0$, it is enough to show that a positive proportion of $n$ have $d$ as their only divisor from $\operatorname{supp}(f)$ and have $n / d$ squarefree. Writing $n=d m$, we observe that these conditions hold unless $m$ belongs to the set of multiples of $\{e / \operatorname{gcd}(e, d): e \neq d, e \in \operatorname{supp}(f)\} \cup\left\{h^{2}: h \geq 2\right\}$. Lemma 2.2 shows that the set of these excluded values of $m$ has density less than 1 , thus completing the proof.

Theorem 1.1 is sharp in the following sense.

Proposition 3.1. Let $Z(x)$ be any increasing function on $[2, \infty)$ that tends to infinity (no matter how slowly). There is a nonzero Möbius pair $(f, g)$ for which

$$
\sum_{\substack{n \leq x \\ n \in \operatorname{supp}(g)}} \frac{1}{n}<Z(x)
$$

for all large $x$, but $\operatorname{supp}(f)$ has asymptotic density zero.

Proof. Let $\mathscr{P}$ be the set of primes constructed greedily by the following process: start with $\mathscr{P}=\emptyset$. Running through the sequence of primes $2,3,5, \ldots$ consecutively, throw $q$ into $\mathscr{P}$ if $\prod_{p \in \mathscr{P} \cap[2, q]}(1+1 /(p-1))<Z(q)$. Since $Z \rightarrow \infty$, the greedy nature of the construction guarantees that $\prod_{p \in \mathscr{P}}(1+1 /(p-1))$ diverges to infinity, 
so that $\sum_{p \in \mathscr{P}} p^{-1}$ diverges. Now let $g$ be the completely multiplicative function with $g(p)=-1$ if $p \in \mathscr{P}$, and $g(p)=0$ otherwise. Let $f(n):=\sum_{d \mid n} g(d)$. If $p \| n$ where $p \in \mathscr{P}$, then $f(n)=f(p) f(n / p)=(1+g(p)) f(n / p)=0$. So, if $n \in \operatorname{supp}(f)$, then there is no prime $p \in \mathscr{P}$ for which $p \| n$. Since $\mathscr{P}$ has divergent reciprocal sum, the set of $n$ satisfying this last condition has density

$$
\prod_{p \in \mathscr{P}}\left(1-\frac{1}{p}+\frac{1}{p^{2}}\right)=0
$$

by an elementary sieve argument (see [Pol09, Lemma 8.13, p. 260]). So $\operatorname{supp}(f)$ has density 0 . On the other hand, if $g(n) \neq 0$, then every prime factor of $n$ belongs to $\mathscr{P}$. So, for large $x$,

$$
\sum_{\substack{n \leq x \\ n \in \operatorname{supp}(g)}} \frac{1}{n} \leq \prod_{\substack{p \leq x \\ p \in \mathscr{P}}}\left(1+\frac{1}{p}+\frac{1}{p^{2}}+\cdots\right)=\prod_{\substack{p \leq x \\ p \in \mathscr{P}}}\left(1+\frac{1}{p-1}\right)<Z(x) .
$$

This completes the proof of the proposition.

It is not hard to prove the analogue of Proposition 3.1 with the roles of $f$ and $g$ reversed; we leave this to the reader.

\section{Proof of Theorem 1.2}

We first suppose that $\sum|g(n)| / n<\infty$ and show that $|f|$ possesses a nonzero mean value. At the end of this section, we discuss the changes necessary to reverse the roles of $f$ and $g$. For each $y \geq 1$, we define an arithmetic function $f_{y}$ by

$$
f_{y}(n):=\sum_{\substack{d \mid n \\ d \leq y}} g(d)
$$

analogously, we define $g_{y}$ by

$$
g_{y}(n):=\sum_{\substack{d \mid n \\ d \leq y}} \mu\left(\frac{n}{d}\right) f(d)
$$

Lemma 4.1. Assume that $\sum_{n=1}^{\infty}|g(n)| / n<\infty$. Then:

(i) for every $y$, the function $\left|f_{y}\right|$ possesses a finite mean value, say $\lambda_{y}$;

(ii) the constants $\lambda_{y}$ tend to a finite limit $\lambda$ as $y \rightarrow \infty$;

(iii) the mean value of $|f|$ is $\lambda$.

Proof. Let $\mathscr{A}$ be the (finite, so also thin) set of natural numbers not exceeding $y$. Since $f_{y}(n)$ depends only on the set of elements of $\mathscr{A}$ dividing $n$, we can write

$$
\sum_{n \leq x}\left|f_{y}(n)\right|=\sum_{\mathscr{S} \subset \mathscr{A}}\left|\sum_{d \in \mathscr{S}} g(d)\right| \sum_{\substack{n \leq x \\ \mathscr{S}=\{d \mid n: d \in \mathscr{A}\}}} 1 .
$$


Dividing by $x$ and letting $x \rightarrow \infty$, we obtain the existence of the mean values $\lambda_{y}$ from Lemma 2.3. (We need only the half of that lemma concerned with prescribing $\mathscr{S}$.) This completes the proof of (i). To see that the $\lambda_{y}$ converge to a finite limit $\lambda$, notice that if $y_{0}<y_{1}$,

$$
\begin{aligned}
\left|\sum_{n \leq x}\right| f_{y_{1}}(n)\left|-\sum_{n \leq x}\right| f_{y_{0}}(n)|| & \leq \sum_{n \leq x}\left|f_{y_{1}}(n)-f_{y_{0}}(n)\right| \\
& \leq \sum_{n \leq x} \sum_{y_{0}<d \leq y_{1}}|g(d)| \\
& \leq x \sum_{d>y_{0}} \frac{|g(d)|}{d} .
\end{aligned}
$$

Dividing by $x$ and letting $x \rightarrow \infty$ shows that

$$
\left|\lambda_{y_{1}}-\lambda_{y_{0}}\right| \leq \sum_{d>y_{0}} \frac{|g(d)|}{d} .
$$

The right-hand side tends to zero as $y_{0} \rightarrow \infty$. Thus, $\left\{\lambda_{y}\right\}$ is a Cauchy net of real numbers, and hence convergent. So, we have (ii). The same arguments used to prove (ii) show that

$$
\left|\sum_{n \leq x}\right| f(n)\left|-\sum_{n \leq x}\right| f_{y_{0}}(n)|| \leq x \sum_{d>y_{0}} \frac{|g(d)|}{d} .
$$

Thus,

$$
\lambda_{y_{0}}-\sum_{d>y_{0}} \frac{|g(d)|}{d} \leq \liminf _{x \rightarrow \infty}\left(\frac{1}{x} \sum_{n \leq x}|f(n)|\right) \leq \limsup _{x \rightarrow \infty}\left(\frac{1}{x} \sum_{n \leq x}|f(n)|\right) \leq \lambda_{y_{0}}+\sum_{d>y_{0}} \frac{|g(d)|}{d} .
$$

Now letting $y_{0} \rightarrow \infty$ gives (iii).

Proof that $|f|$ HaS a NonZero mean value, assuming $\sum_{n=1}^{\infty}|g(n)| / n<\infty$. It remains only to show that the number $\lambda$ from Lemma 4.1 is positive. Let $d$ be the smallest element of the support of $g$. We consider the partial sums of $|f|$ restricted to $n$ of the form $d m$, where every prime dividing $m$ exceeds a large but fixed real parameter $y$. Using ' to denote this restriction,

$$
\begin{aligned}
\sum_{n \leq x}^{\prime}|f(n)| & \geq \sum_{n \leq x}^{\prime}\left(|g(d)|-\sum_{\substack{e \mid n \\
e \neq d}}|g(e)|\right) \\
& =\left(|g(d)| \sum_{n \leq x}^{\prime} 1\right)-\sum_{n \leq x}^{\prime} \sum_{\substack{e \mid n \\
e \neq d}}|g(e)| .
\end{aligned}
$$


We proceed to estimate the remaining sums. If we put $P:=\prod_{p \leq y} p$, then

$$
\begin{aligned}
\sum_{n \leq x}^{\prime} 1 & =\sum_{\substack{m \leq x / d \\
\operatorname{gcd}(m, P)=1}} 1 \\
& =\frac{x}{d} \prod_{p \leq y}\left(1-\frac{1}{p}\right)+O\left(2^{\pi(y)}\right),
\end{aligned}
$$

where the last step follows by inclusion-exclusion (for example, see [MV07, Theorem 3.1, p. 76]). Observe that if $n=d m$, where all of the prime factors of $m$ exceed $y$, then every divisor $e$ of $n$ belongs to $[1, d]$ or has a prime divisor greater than $y$. So, if $e \neq d$, then the choice of $d$ forces $g(e)=0$ or $e>y$. Hence (applying inclusion-exclusion once again),

$$
\begin{aligned}
\sum_{n \leq x}^{\prime} \sum_{\substack{e \mid n \\
e \neq d}}|g(e)| & \leq \sum_{e>y}|g(e)| \sum_{\substack{n \leq x \\
e \mid n}}^{\prime} 1 \leq \sum_{e>y}|g(e)| \sum_{\substack{m \leq x / \operatorname{lcm}(d, e) \\
p \mid m \Rightarrow p>y}} 1 \\
& \leq x \prod_{p \leq y}\left(1-\frac{1}{p}\right)\left(\sum_{e>y} \frac{|g(e)|}{\operatorname{lcm}(d, e)}\right)+O\left(2^{\pi(y)} \sum_{y<e \leq x}|g(e)|\right) .
\end{aligned}
$$

Since $\sum_{n \geq 1}|g(n)| / n<\infty$, Lemma 2.4 guarantees that the final error term in (4.3) is $o(x)$, as $x \rightarrow \infty$. Now we substitute (4.3) and (4.2) back into (4.1), divide by $x$, and let $x \rightarrow \infty$ to find that

$$
\begin{aligned}
\liminf _{x \rightarrow \infty} \frac{1}{x} \sum_{n \leq x}^{\prime}|f(n)| & \geq\left(\frac{|g(d)|}{d}-\sum_{e>y} \frac{|g(e)|}{\operatorname{lcm}(d, e)}\right) \prod_{p \leq y}\left(1-\frac{1}{p}\right) \\
& \geq\left(\frac{|g(d)|}{d}-\sum_{e>y} \frac{|g(e)|}{e}\right) \prod_{p \leq y}\left(1-\frac{1}{p}\right) .
\end{aligned}
$$

But if $y$ is fixed sufficiently large, then this last expression is positive. Since $\sum_{n \leq x}|f(n)| \geq \sum_{n \leq x}^{\prime}|f(n)|$, it must be that the mean value $\lambda$ of $|f|$ is positive.

We now consider the effect of swapping $f$ and $g$. That the mean value of $|g|$ exists if $\sum_{n=1}^{\infty}(|f(n)| / n)<\infty$ follows by the same arguments used to prove Lemma 4.1, after swapping $f$ and $g$. There is only one substantial change necessary: the value of $f_{y}(n)$ depends both on the set of divisors $d$ of $n$ not exceeding $y$ and on the subset of those $d$ for which $n / d$ is squarefree. So we must appeal to the full force of Lemma 2.3. We leave the remaining details to the reader.

Finally, we show that the mean value of $|g|$ is positive.

Proof that $|g|$ Has a nonzero mean value, assuming $\sum_{n=1}^{\infty}|f(n)| / n<\infty$. We let $d$ be the least element of the support of $f$. We let $y$ denote a large but fixed real parameter, and we use ' with the same meaning as before. The reasoning that led us to (4.1) now shows that

$$
\sum_{n \leq x}^{\prime}|g(n)| \geq|f(d)| \sum_{n \leq x}^{\prime}\left|\mu\left(\frac{n}{d}\right)\right|-\sum_{n \leq x}^{\prime} \sum_{\substack{e \mid n \\ e \neq d}}|f(e)| .
$$


Following our earlier proof, we obtain our previous upper bound (4.3) for the double sum here, except now with $g$ replaced by $f$. Appealing again to Lemma 2.4 and using that $\operatorname{lcm}(d, e) \geq e$, we thus see that

$$
\sum_{n \leq x}^{\prime} \sum_{\substack{e \mid n \\ e \neq d}}|f(e)| \leq x \prod_{p \leq y}\left(1-\frac{1}{p}\right) \sum_{e>y} \frac{|f(e)|}{e}+o(x),
$$

as $x \rightarrow \infty$. On the other hand, one has that, as $x \rightarrow \infty$,

$$
\begin{aligned}
\sum_{n \leq x}^{\prime}\left|\mu\left(\frac{n}{d}\right)\right| & =\sum_{\substack{m \leq x / d \\
m \text { squarefree } \\
p \mid m \Rightarrow p>y}} 1 \\
& \sim \frac{6}{\pi^{2}} \cdot \frac{x}{d} \prod_{p \leq y}\left(1+\frac{1}{p}\right)^{-1},
\end{aligned}
$$

by Lemma 2.5 with $P:=\prod_{p \leq y} p$. Substituting (4.7) and (4.6) back into (4.5), dividing by $x$, and letting $x \rightarrow \infty$,

$$
\begin{aligned}
\liminf _{x \rightarrow \infty} \frac{1}{x} \sum_{n \leq x}^{\prime}|g(n)| & \geq \frac{6}{\pi^{2}} \cdot \frac{|f(d)|}{d} \prod_{p \leq y}\left(1+\frac{1}{p}\right)^{-1}-\prod_{p \leq y}\left(1-\frac{1}{p}\right) \sum_{e>y} \frac{|f(e)|}{e} \\
& \geq\left(\prod_{p \leq y}\left(1-\frac{1}{p}\right)\right)\left(\frac{6}{\pi^{2}} \cdot \frac{|f(d)|}{d}-\sum_{e>y} \frac{|f(e)|}{e}\right) .
\end{aligned}
$$

This final expression is positive if $y$ is fixed sufficiently large. So the mean value of $|g|$ must be positive.

Remark 4.2. Suppose that $(f, g)$ is a Möbius pair with $\sum_{n \geq 1}|g(n)| / n<\infty$. We showed above that the mean value of $|f|$ must be positive. That proof in fact shows that if every element in $\operatorname{supp}(g)$ is at least $d$, and $y \geq 1$, then

$$
\liminf _{x \rightarrow \infty} \frac{\sum_{n \leq x}^{\prime}|f(n)|}{\sum_{n \leq x}^{\prime} 1} \geq|g(d)|-d \sum_{e>y} \frac{|g(e)|}{e} .
$$

Here, as before, $\Sigma^{\prime}$ denotes a sum restricted to integers $n$ of the form $d m$, where each prime factor of $m$ exceeds $y$. (Compare the asserted inequality with (4.4).) A completely analogous argument shows that under the same hypotheses,

$$
\limsup _{x \rightarrow \infty} \frac{\sum_{n \leq x}^{\prime}|f(n)|}{\sum_{n \leq x}^{\prime} 1} \leq|g(d)|+d \sum_{e>y} \frac{|g(e)|}{e} .
$$

Here is an amusing application of (4.8), in the spirit of [Pol11]. Suppose for the sake of contradiction that the sum of the reciprocals of the prime numbers converges. Take $g$ 
to be the characteristic function of the primes, take $d=1$, and take $y$ large enough that $\sum_{p>y} 1 / p<1$. Since $f(n)=\sum_{p \mid n} 1$ counts the number of distinct primes dividing $n$, we have $f(n) \geq 1$ for all $n>1$, and so the left-hand side of (4.8) is at least 1. But the right-hand side is smaller than 1 , a contradiction!

\section{Proof of Theorem 1.4}

We first deal with the case when $\beta<1$. Fix disjoint sets of primes $\mathscr{P}_{0}$ and $\mathscr{Q}_{0}$ with $\prod_{p \in \mathscr{P}_{0}}(1-1 / p)=\prod_{p \in \mathscr{Q}_{0}}(1-1 / p)=0$. We can find a subset $\mathscr{P} \subset \mathscr{P}_{0}$ so that $\prod_{p \in \mathscr{P}}(1-1 / p)=\alpha$, where the minimum of $\mathscr{P}$ is so large that $\prod_{p \in \mathscr{P}}\left(1-1 / p^{2}\right) \geq \beta$. We can then select a set $\mathscr{Q} \subset \mathscr{Q}_{0}$ so that

$$
\prod_{p \in \mathscr{Q}}\left(1-\frac{1}{p}\right)=\beta \prod_{p \in \mathscr{P}}\left(1-\frac{1}{p^{2}}\right)^{-1} .
$$

Define $g$ as the multiplicative arithmetic function whose values at prime powers are given by

$$
g\left(p^{e}\right):=\left\{\begin{aligned}
-1 & \text { if } p \in \mathscr{P} \text { and } e=1, \\
0 & \text { if } p \in \mathscr{P} \text { and } e>1, \\
0 & \text { if } p \in \mathscr{Q} \\
+1 & \text { if } p \notin \mathscr{P} \cup \mathscr{Q} .
\end{aligned}\right.
$$

Then the function $f(n):=\sum_{d \mid n} g(d)$ is multiplicative, and after computing $f$ at prime powers, we see that $\operatorname{supp}(f)$ consists of all the positive integers without prime factors from $\mathscr{P}$. Now an easy sieve argument (compare with [Pol09, Corollary 6.3, p. 173]) shows that

$$
\mathbf{d}(\operatorname{supp}(f))=\prod_{p \in \mathscr{P}}\left(1-\frac{1}{p}\right)=\alpha .
$$

Moreover, the support of $g$ consists of those positive integers without prime factors in $\mathscr{Q}$, and which are not divisible by any $p^{2}$ with $p \in \mathscr{P}$. Another simple sieve argument now shows that

$$
\mathbf{d}(\operatorname{supp}(g))=\prod_{p \in \mathscr{Q}}\left(1-\frac{1}{p}\right) \prod_{p \in \mathscr{P}}\left(1-\frac{1}{p^{2}}\right)=\beta .
$$

This completes the proof when $\beta<1$.

Consider now the case in which $\beta=1$. This time, we select $\mathscr{P}$ as any set of prime numbers with $\prod_{p \in \mathscr{P}}\left(1-1 / p+1 / p^{2}\right)=\alpha$; this is possible since $\prod_{p}(1-1 / p+$ $\left.1 / p^{2}\right)=0$. Define $g$ as the multiplicative arithmetic function satisfying

$$
g\left(p^{e}\right):= \begin{cases}-1 & \text { if } p \in \mathscr{P} \text { and } e=1 \\ +1 & \text { if } p \in \mathscr{P} \text { and } e>1 \\ +1 & \text { if } p \notin \mathscr{P} .\end{cases}
$$


Let $f(n):=\sum_{d \mid n} g(d)$. Then $f$ is multiplicative, and computing its values at prime powers, we find that $f(n)=0$ if and only if $p \| n$ for some $p \in \mathscr{P}$. Again, a sieve argument gives

$$
\mathbf{d}(\operatorname{supp}(f))=\prod_{p \in \mathscr{P}}\left(1-\frac{1}{p}+\frac{1}{p^{2}}\right)=\alpha .
$$

Since $g$ is always nonzero, we also have $\mathbf{d}(\operatorname{supp}(g))=1=\beta$.

\section{Concluding remarks}

Call a class of subsets of $\mathbf{N}$ exclusive if for every nonzero Möbius pair $(f, g)$, at least one of the sets $\operatorname{supp}(f)$ and $\operatorname{supp}(g)$ falls outside of the class. As remarked in the introduction, our Theorem 1.1 shows that the thin sets comprise an exclusive class.

We can do a little better than this. Call $\mathscr{A} \subset \mathbf{N}$ evaporating if, as $T \rightarrow \infty$, the upper density of the set of $n$ with a divisor in $\mathscr{A} \cap[T, \infty)$ tends to zero. It is easy to prove that every thin set is evaporating. However, there are evaporating sets that are not thin; an interesting example, due to Erdős and Wagstaff [EW80, Theorem 2], is the set of shifted primes $\{p-1\}$. We can show that the evaporating sets form an exclusive class. In fact, our Theorem 1.1 remains true if we replace the hypothesis that $\operatorname{supp}(g)$ is thin with the assumption that $\operatorname{supp}(g)$ is evaporating. The proof requires relatively minor modifications; the most significant of these is the replacement of Lemma 2.2 with its analogue for evaporating sets (see, for example, [PS88, Lemma 2]).

It would be interesting to know other classes of sets that are also exclusive. The following question seems attractive and difficult.

Question. For each $\delta>0$, consider the class $\mathfrak{C}(\delta)$ of subsets $\mathscr{A} \subset \mathbf{N}$ for which

$$
\#\{n \leq x: n \in \mathscr{A}\} \leq C_{\mathscr{A}} \frac{x}{(\log x)^{\delta}} \quad \text { for some real } C_{\mathscr{A}} \text {, and all } x \geq 2 .
$$

Is this class exclusive?

From Theorem 1.1 and partial summation, $\mathfrak{C}(\delta)$ is exclusive for $\delta>1$. Theorem 1.4 shows that $\operatorname{supp}(f)$ and $\operatorname{supp}(g)$ can both have density zero; taking $\mathscr{P}=\mathscr{P}_{0}=\{p \equiv 1$ $(\bmod 3)\}$ and $\mathscr{Q}=\mathscr{Q}_{0}=\{p \equiv-1(\bmod 3)\}$ in the proof of that theorem, one can show that $\mathfrak{C}(\delta)$ is not exclusive for $\delta \leq \frac{1}{2}$. What about the range $\frac{1}{2}<\delta \leq 1$ ?

\section{Acknowledgement}

We thank Enrique Treviño for suggestions that improved the readability of the paper.

\section{References}

[Erd34] P. Erdôs, 'On the density of abundant numbers', J. Lond. Math. Soc. 9 (1934), 278-282.

[EW80] P. Erdôs and S. S. Wagstaff Jr, 'The fractional parts of the Bernoulli numbers', Illinois J. Math. 24 (1980), 104-112. 
[Ha196] R. R. Hall, Sets of Multiples, Cambridge Tracts in Mathematics, 118 (Cambridge University Press, Cambridge, 1996).

[Hei37] H. A. Heilbronn, 'On an inequality in the elementary theory of numbers', Proc. Cambridge Philos. Soc. 33 (1937), 207-209.

[Kro87] L. Kronecker, 'Quelques remarques sur la détermination des valeurs moyennes', C. R. Acad. Sci. Paris 103 (1887), 980-987.

[Lan53] E. Landau, Handbuch der Lehre von der Verteilung der Primzahlen. 2 Bände, 2nd edn. (Chelsea Publishing Co., New York, 1953).

[MV07] H. L. Montgomery and R. C. Vaughan, Multiplicative Number Theory. I. Classical Theory, Cambridge Studies in Advanced Mathematics, 97 (Cambridge University Press, Cambridge, 2007).

[Pol09] P. Pollack, Not Always Buried Deep (American Mathematical Society, Providence, RI, 2009).

[Pol11] P. Pollack, 'The Möbius transform and the infinitude of primes', Elem. Math. 66 (2011), $118-120$.

[PS88] C. Pomerance and A. Sárközy, 'On homogeneous multiplicative hybrid problems in number theory', Acta Arith. 49 (1988), 291-302.

[Roh37] H. Rohrbach, 'Beweis einer zahlentheoretischen Ungleichung', J. reine angew. Math. 177 (1937), 193-196.

[Win43] A. Wintner, Eratosthenian Averages (Waverly Press, Baltimore, 1943).

PAUL POLLACK, Department of Mathematics, University of Georgia, Athens, Georgia 30602, USA

e-mail:pollack@uga.edu

CARLO SANNA, Department of Mathematics, Università degli Studi di Torino, Turin, Italy

e-mail: carlo.sanna.dev@gmail.com 